\title{
El gobierno en tiempos tempestuosos. Escenas de la democracia ateniense y su relación con la crisis
}

\section{Government during Stormy Times. Scenes of Athenian Democracy and its Connection with the Crisis}

\section{Claudia Mársico ${ }^{1}$ \\ Universidad de Buenos Aires (Argentina)}

ORCID: https://orcid.org/0000-0002-6988-9333

Recibido: 01-06-2020

Aceptado: 21-06-2020

\section{Resumen}

El presente trabajo está orientado al análisis de la relación entre democracia y crisis prestando atención a los antecedentes ajenos al contexto griego y el desarrollo del caso ateniense. Sobre esa base, se revisan dos tipos de fricción con otros modelos políticos. Por un lado, los elementos de tensión con la aristocracia, a través del testimonio de Tucídides y del Viejo Oligarca, y con los gobiernos unipersonales, a través del Hierón de Jenofonte, a los efectos de evaluar el papel de la relación con la crisis en su perfil general.

Palabras-clave: democracia, aristocracia, crisis, conflicto.

\footnotetext{
${ }^{1}$ (claudiamarsico@conicet.gov.ar) Claudia Marsico es Profesora Titular de Filosofía Antigua en la Universidad de Buenos Aires e Investigadora Titular del Consejo Nacional de Investigaciones Científicas y Tecnológicas (CONICET) de Argentina. Actualmente es Directora de la Sección de Filosofía Antigua de la Academia Nacional de Ciencias de Buenos Aires. Es la presidenta fundadora de la Sociedad Internacional de Estudios Socráticos y miembro de pleno derecho de la Sociedad Internacional Platón, entre otros. Ha dirigido numerosos proyectos internacionales, becas, maestrías y doctorados y participado en numerosos eventos académicos regionales e internacionales en Alemania, España, Francia, Inglaterra, Irlanda, Israel, Italia, Serbia, Estados Unidos y América Latina. Cuenta con numerosas publicaciones en su campo, entre ellas la traducción de los testimonios existentes de los filósofos socráticos (Madrid, Losada, 2013-2014, 2 vols.), La primera versión integral a un lenguaje moderno, así como las cartas de Sócrates y los socráticos (Buenos Aires, Miluno, 2012), y el capítulo sobre Esquines en el volumen colectivo internacional sobre Sócrates y el diálogo socrático editado por A. Stavru y C. Moore (Leiden, Brill, 2017), entre muchos otros.
} 


\begin{abstract}
This paper is oriented to the analysis of the relationship between democracy and crisis, paying attention to the antecedents outside the Greek context and its development in Athens. On this basis, it reviews two kinds of friction with other political models. On the one hand, the elements of tension with aristocracy, through the testimony of Thucydides and the Old Oligarch, and with the governments run by one person, through Xenophon's Hiero, to assess the role of the relationship with crisis in the general profile.
\end{abstract}

Key-words: Democracy, Aristocracy, Crisis, Conflict.

La cita del título es enunciada por el Sócrates platónico de República con ironía. Como sucede con las cosas del cambiante plano sensible, es bella para unos y no para otros. La celebran las mujeres y los niños, que se deleitan con objetos coloridos. No se trata, es claro, de las guardianas y sus hijos comunes como señal de la superación de todo lo dado, sino de las atenienses y sus vástagos que ni siquiera participan de los asuntos políticos. Desde la mirada del filósofo platónico, por debajo de ella sólo queda la tiranía. Estamos, en efecto, lejos de las nociones modernas de democracia y el tipo de discusiones que atraviesan el s. IV a.C. se asocia con las fricciones peculiares de la democracia, la oligarquía y los gobiernos unipersonales de esa época. Sin embargo, tanto en la evaluación platónica como en otras de la época se reiteran las alusiones a la situación de crisis. Para comprender este sustrato es necesario remontarse al modo en que este modelo de gobierno se instauró en Atenas. Para ello, analizaremos primero los elementos proto-democráticos que se encuentran en la tradición antigua y prefiguran, no siempre con rasgos coincidentes, el modelo que surgirá en Atenas. En este punto, nos interesa examinar la relación entre democracia y crisis, a los efectos de subrayar sus puntos de contacto. Sobre esta base, revisaremos dos tipos de fricción de la democracia con otros sistemas políticos en las primeras reflexiones sobre la democracia: con la aristocracia, a través del testimonio de Tucídides y del Viejo Oligarca, y con los gobiernos unipersonales, a través del Hierón de Jenofonte. Este recorrido echará luz sobre el tipo de fenómeno que está en la base de las discusiones teóricas de la época clásica.

\title{
1. La prehistoria de la democracia
}

La crisis ateniense está asociada a la crisis de la democracia, producto que pasa por ser de pura factura griega. Sin embargo, los indicios de organizaciones democráticas o proto-democráticas en culturas más antiguas cercanas al 
mediterráneo se han venido extendiendo los últimos años de manera sostenida. La primera clave para la comprensión de la crisis ateniense del s. V a.C. está conectada con el tipo de modelo político en juego y lo primero que habría que cuestionar es la lectura que la reduce a las resistencias y desequilibrios asociados con el inicio. En el caso de la Mesopotamia, en las ciudades del sur asociadas con Sumer, hay testimonios del funcionamiento habitual de una asamblea, el ukkin - puhrum para los acadios-, aunque hay pocos datos taxativos sobre el alcance de su poder y los criterios de elección de sus miembros. Con algo de exageración se ha llamado "sistema bicameral" a los cuerpos de ancianos y jóvenes que condicionaban las decisiones del rey en el relato de Gilgamesh y Agga, que se remonta al 2700 a.C. ${ }^{2}$. La versión ninivita que se ubica en torno al s. VII a.C. conserva el tópico, lo cual indicaría la persistencia de esta práctica.

Esto no es de extrañar, ya que los reyes de Ur del inicio de comienzos del tercer milenio a.C. dependían de una asamblea, incluso para su designación, con organización interna y multiplicidad de funciones. Si se recuerda que el imaginario señala dioses que toman decisiones por consenso a partir de reuniones, toma forma una suerte de democracia primitiva que preanuncia muchos rasgos de la que luego se desarrollaría en Grecia. Asimismo, la asamblea es una institución en el código de Hamurabi hasta el punto de que ante una situación de vacío de poder debe hacerse cargo del gobierno además de cumplir con sus funciones judiciales ${ }^{3}$. Hay relatos sobre jurados con participación de trabajadores y algunos infieren incluso la presencia de mujeres, en un concierto que enlaza las tradiciones democráticas con las ciudades más antiguas a la manera de una anticipación del "invento" griego.

En algún sentido, también hay elementos proto-democráticos en la tradición hebrea y su asamblea de Israel que ratificaba el derecho al trono y aseguraba la consulta y el consenso. No es improbable que estas instituciones se remonten precisamente a las épocas del exilio Babilonia, hacia el s. VI a.C. ${ }^{4}$. Incluso Egipto, al que se acude habitualmente como encarnación del caso opuesto, puede mirarse bajo otra luz. Atendiendo a los rasgos de un sistema complejo, el faraón cumple un papel importante, pero también ahí funciona una asamblea responsable de la administración de pueblos que se reserva funciones judiciales ${ }^{5}$. En comparación con las mesopotámicas, las asambleas egipcias resultan restringidas y formadas básicamente por notables, pero no deja de ser curioso que la abolición de los consejos ciudadanos se haya dado en la época de los gobiernos griegos de los Ptolomeos.

Fenicia, finalmente, de donde los griegos toman el alfabeto, presenta un sistema que combina democracia y oligarquía en la composición de asambleas

\footnotetext{
${ }^{2}$ Véase Martin -Snell (2005, pp. 397-407) y Glassman (2017, pp. 303-324).

3 Véase Schemeil (2000, pp. 99-120) y Van De Mieroop (2013, pp. 277-89).

${ }^{4}$ Véase Jacobsen (1943, pp. 159-172), Wolf (1947, pp. 98-108) y Gottwald (2001).

${ }^{5}$ Véase Schemeil (2000, pp. 99-120) y Isakhan (2007).
} 
con poder en el gobierno de las ciudades y colonias. El Informe de Wen-Amin, un texto egipcio de 1000 a.C., relata las dificultades para tratar comercialmente con los fenicios por la cantidad de cuerpos y agentes intermedios de decisión ${ }^{6}$. Junto con el alfabeto, en algún sentido Fenicia puede haber legado ideas políticas a una prehistoria europea que continuaría su historia combinando gobiernos de ricos y representación de grupos influyentes hasta la actualidad.

Este amplio abanico de modelos ilustra, entonces, la presencia de instituciones de representación, pero en general sometidas al primado de monarquías u oligarquías muchas veces en tensión, que no dejaban lugar al crecimiento de quienes encarnan la base de esta tercera alternativa. Lo que aporta el caso griego, entonces, no es originalidad en la creación de un modelo, sino un debilitamiento de monarquía y oligarquía que permitieron un despliegue sin precedentes de los parámetros democráticos. Esta tríada se remonta a Heródoto y su relato sobre la evaluación de los modos de gobierno en III.80-82 donde se evalúan las ventajas y desventajas de la monarquía, la aristocracia y la democracia. Lo que hemos dicho sobre la democracia antes y fuera de Atenas aligera bastante la línea de lecturas que juzga casi un sinsentido proyectar en la corte de Darío la discusión sobre este punto.

La proyección en terreno persa puede verse probablemente como un exponente de la tradición que marcó Harold Cherniss respecto de la tendencia griega de proyectar todo lo nuevo como factor importado y evitar cualquier explicación que requiera una explicación de transformación local que dé cuenta de la innovación ${ }^{7}$. La aplicación al propio terreno, no obstante, es clara. Mutatis mutandis, los rasgos de los tipos políticos de Heródoto no son controvertidos en lo central por las caracterizaciones directamente dirigidas a pensar la crisis ateniense. Más aún, las colisiones que se dan entre la democracia y sus modos rivales ofrecen elementos interesantes para comprender sus condicionamientos.

Conviene recordar que 'crisis' incluye, por mandato etimológico, su relación con el "criterio", en el sentido de la evaluación del juicio más adecuado sobre una $\operatorname{cosa}^{8}$. Pero contiene un sentido que, fortalecido a lo largo de la historia, advierte la disrupción interna del krínein. Al cernir sacudiendo para separar lo mezclado se fracturan los compuestos y lo que se veía unido resulta expuesto en su multiplicidad. El escenario de la democracia en crisis no encarna el orden racional que enfrenta resistencias, sino en todo caso la experiencia de la ampliación de la base de agentes de toma de decisiones en un contexto permeable a una inestabilidad constitutiva llamativa muy propia de la tradición que se inicia con los griegos y configura una de sus grandes pervivencias a lo largo de la historia posterior. Lo que se cierne y se criba

\footnotetext{
${ }^{6}$ Véase Stockwell (2011, pp. 35-48).

${ }^{7}$ Véase Cherniss (1953, pp. 22-47).

${ }^{8}$ Véase Chantraine (1968, s.v. krino).
}

Araucaria. Revista Iberoamericana de Filosofia, Politica, Humanidades y Relaciones Internacionales, año $23, \mathrm{n}^{\circ} 46$. Primer cuatrimestre de 2021. Pp. 71-84. ISSN 1575-6823 e-ISSN 2340-2199 https://dx.doi.org/10.12795/araucaria.2021.i46.04 
son precisamente los modelos políticos de gobierno a la mano haciéndolos colisionar, diseccionándolos y recomponiéndolos con elementos producto de la mixtura.

Aun en tiempos recientes el relato sobre el milagro griego y su prístina racionalidad tiñe la interpretación de estos procesos, sugiriendo que la democracia era un producto resultante del debilitamiento de los otros modelos que operaría como una suerte de superación. En esta línea, el proceso encabezado por Clístenes se apoyaría en una renuncia voluntaria de los grupos en pugna que funda un rediseño cuasi-matemático ${ }^{9}$. Por esta vía se ideó una oposición de lo griego frente a lo no griego, se polarizaron los rasgos y democracia y razón quedaron del primer lado, frente a los influjos tiránicos e irracionales que configuraron la barbarie. Los problemas comienzan cuando esta supuesta asociación, que debería suponer orden, colisiona con la crisis, que se reitera con una persistencia demasiado marcada como para pretender que adviene por factores externos. El retorno de formas oligárquicas y monárquicas conspira, por supuesto, contra la idea de una superación y su carácter problemático se refleja en múltiples dominios culturales del mundo griego, incluyendo la filosofía, la literatura y la historiografía

Notemos dos puntos por los cuales esta vía no es teóricamente productiva. En primer lugar, el imaginario griego provee un horizonte más complejo e irreductible a los relatos de superación. Sugiere, por el contrario, sustratos de largo término que se proyectan incluso más allá de lo griego y emergen en nuestra tradición en momentos en que las ideas-fuerza de los períodos "estables" se resquebrajan, ya se trate de Edad Media y Dios, tras lo cual no es casual la vuelta de la iconografía clásica, o se trate de la razón moderna, que deja advenir una contemporaneidad profundamente griega en su sensación de extravío. En efecto, el imaginario griego propone una religiosidad sin dogmas, que no le dice al hombre cómo se debe vivir y tampoco le indica cómo convivir ${ }^{10}$. La política divina es, por tanto, un preludio de los avatares que despiertan la reacción de los autores de la época clásica. En lo político, lo divino ofrece un modelo de politeísmo jerárquico y conflictivo. Zeus gobierna, pero sobre un suelo inestable, en una suerte de thriller político primitivo lleno de monarcas derrocados y engañados.

El segundo punto a notar es que lo divino es un punto intermedio en la cadena de condicionamientos, con la Necesidad por encima y por debajo al hombre, que resulta así un eslabón con capacidad de decisión disminuida, como muestran los ejemplos de la disculpa de Agamenón, o la sobredeterminación de las muertes rematadas por dioses. Dioses en tal tembladeral sólo pueden lanzar mensajes contradictorios, porque estrictamente no manejan la

\footnotetext{
${ }^{9}$ Véase Rodríguez Guerra (2011, pp. 149-168).
}

${ }^{10}$ Véase Mársico (2011). 
ultima ratio. Ya sea que esto provoque en los hombres una situación de angustia y desconcierto o, al contrario, la aceptación heroica ante un destino inmanejable, podríamos decir que se trata variantes de la respuesta ante lo que actualmente consideramos una crisis, enfatizando precisamente el aspecto de resquebrajamiento y desmembramiento de lo previo, que en esta perspectiva no resulta un acontecimiento inesperado sino el motor del relato cósmico que lega el pensamiento griego. Incluso los sistemas que apuestan a elementos estables, lo hacen en lucha contra lo indómito como dato primario de nuestra experiencia.

Imaginario cultural y crisis resultan de este modo aspectos coextensivos. La democracia no es un orden frágil que se rompe, sino una de las figuras de una inmanencia dinámica instalada sobre la inestabilidad, la dilución y la transformación, que no resultan una anomalía, sino lo más cercano a una persistencia en su intrínseca diferencia. No es menor que una buena parte de las reflexiones historiográficas contemporáneas partan de la constatación de la variación del sentido de la noción de democracia y deriven de allí consecuencias asociadas con la mutación de las nociones, como sucede paradigmáticamente en las líneas asociadas con la Historia Conceptual ${ }^{11}$. Tampoco es casual que las numerosas corrientes contemporáneas preocupadas por la noción de diferencia vuelvan con renovado ímpetu a buscar en los resquicios de Grecia sus preludios.

La crisis de la democracia griega del s. V a.C. es, por tanto, un caso dentro dentro de una tendencia general con marcadas variantesl que afecta muy especialmente las coordenadas del pensamiento griego. Esto claramente no significa que no haya crisis e inestabilidad en otras culturas, pero sí que este rasgo está enraizado en Grecia de modo especialmente marcado, donde la crisis permanentemente "granula" y tamiza lo real dejándolo como materia presta para configuraciones siempre nuevas. En este contexto la crisis es motor de destrucción pero también de revisión de lo actual y creación de lo nuevo, en una intuición que fulgura desde los poéticos dichos de Anaximandro, no porque haya ciclos, sino porque la dinámica de transformación lo afecta todo, incluyendo las creaciones conceptuales que intentan aferrarse a una estabilidad en lo inteligible. Desde la aceleración de los cambios que Koselleck asocia con la modernidad hasta la época del Gestell heideggeriano están prefigurados en los influjos del krínein que sacuden todo sin preservar puntos fijos.

\section{La fricción con otros tipos de gobierno}

Dicho esto, podemos concentrarnos en dos aspectos de la configuración de la crisis ateniense que muestran este diseño y traen aparejadas precisamente

11 Véase Vilanou (2006, pp. 165-190).

Araucaria. Revista Iberoamericana de Filosofia, Politica, Humanidades y Relaciones Internacionales, año $23, \mathrm{n}^{\circ} 46$. Primer cuatrimestre de 2021. Pp. 71-84. ISSN 1575-6823 e-ISSN 2340-2199 https://dx.doi.org/10.12795/araucaria.2021.i46.04 
las primeras reflexiones sobre el fenómeno de la democracia. Habiendo mencionado la síntesis de Heródoto y los tres sistemas de gobierno, revisemos los ejes de la colisión que experimenta la democracia en la crisis del s. V a.C. comenzando por la fricción con la oligarquía. La selección algo aleatoria de la tradición nos ha legado el agón entre el testimonio de Tucídides, que en su cuantioso material cuenta con los discursos atribuidos a Pericles que encarnan una defensa de la democracia, y el contrapunto ofrecido por la Constitución de los atenienses del Viejo Oligarca.

En el primer discurso de Pericles Tucídides retrata el clima de 432 a.C. y la apertura de hostilidades contra Esparta. Allí Pericles avanza en un estudio de las condiciones de los adversarios que constituye un breve estudio de la organización política espartana, donde se resalta que trabajan ellos mismos la tierra, tienen riquezas comunalizadas sin uso de dinero, se valen de maniobras terrestres que resultan costosas y difíciles y, si bien son poderosos, no manejan bien a sus aliados (I.141.2-7). Este análisis da ocasión para constatar que el gobierno del mar es ateniense y eso debería permitirles aventurarse en el conflicto (I.142.1-9). El Viejo Oligarca reitera la descripción desde la perspectiva de los que sufren el poder del mar en manos ajenas (II.2-6 y II.1113) y alude al abandono estratégico del territorio circundante que permite que sea arrasado sin poner en riesgo la ciudad (II.14). Del mismo modo Tucídides retrata a Pericles recomendando el repliegue en la ciudad fortificada (I.143.5), en la estrategia que provocó el hacinamiento de la población y provocó la peste que arrancó la vida de numerosos habitantes, incluido el mismo Pericles.

Los ecos retornan en el discurso fúnebre. La estrategia de honra a los caídos en el primer año de la Guerra se convierte en un espacio para un ejercicio reflexivo sobre las virtudes cívicas de Atenas. El género de epitháphios lógos es en sí mismo un producto propio de esta comunidad que cataliza el duelo privado y lo transforma en expresión de ideología común precisamente en momentos donde la crisis lo vuelve necesario ${ }^{12}$. Constituye, en este sentido, una instancia privilegiada para reflexionar sobre la identidad de Atenas y ligarla al compromiso con la democracia en crisis. Tucídides retrata a Pericles en esta tarea, insistiendo primero en la originalidad de la organización política ateniense que no imita otros modelos (II.37.1). Los partidarios de la oligarquía, por el contrario, son acusados de emular a Esparta no como ejemplo de un modelo político posible sino como agentes pro-extranjeros, en una dinámica extendida en todas las épocas.

\footnotetext{
${ }^{12}$ Estas oraciones fúnebres se celebraban anualmente durante períodos de guerra, acompañadas de procesiones hasta la tumba del Cerámico donde se pronunciaba la alocución, y contienen, por tanto, elementos marcadamente agonísticos que ensalzan a la ciudad dejando en segundo plano al orador y a los caídos, fundiendo en un solo tópico el destino de la ciudad y la bella muerte que en el hombre se plasma en el sacrificio por la patria. Véase Loraux (1986) y Derderian (2001: $161 \mathrm{ss).}$
}

Araucaria. Revista Iberoamericana de Filosofia, Política, Humanidades y Relaciones Internacionales, año $23, \mathrm{n}^{\circ} 46$. Primer cuatrimestre de 2021. Pp. 71-84. ISSN 1575-6823 e-ISSN 2340-2199 https://dx.doi.org/10.12795/araucaria.2021.i46.04 
En este texto cobra especial importancia, al mismo tiempo, la respuesta de Pericles a las acusaciones de promoción de los inútiles en términos de una igualdad que hace lugar al mérito personal y no a cuestiones de clase, por lo cual "nadie, en razón de su pobreza, encuentra obstáculos debido a la oscuridad de su condición social" (II.37.1). Lo que el Viejo Oligarca ve como impulso a los peores, Pericles lo plantea como verdadero respaldo a los mejores (II.37.2). Con la misma lógica, Pericles declara una obediencia sostenida a las leyes (II.37.3) que hace de Atenas un ejemplo para Grecia y de cada ateniense una persona sobresaliente, de lo cual es prueba el poderío de la ciudad (II.41.1-2).

Los mismos fenómenos son evaluados desde escalas axiológicas diversas y dan por resultado la colisión de imaginarios que caracteriza el krínein al que hemos aludido. Donde Tucídides despliega un demócrata encendido, el Viejo Oligarca avanza mirando desde el otro lado los desequilibrios que produce un sistema así y todo efectivo. En efecto, el autor se dirige a un interlocutor que desprecia el modelo ateniense y es esta supuesta actitud la que dicta una estrategia doble: supone primero un acercamiento a la posición anti-democrática apelando a una serie de lugares comunes sobre las fallas de su organización básica, para avanzar luego en una explicación acumulativa de aspectos que revelan la racionalidad intrínseca del sistema y el modo en que cada una de sus conductas previstas lo fortalece. De este modo, donde una posición menos sutil sólo ve bestialidad e ignorancia, la Constitución de los atenienses advierte intereses de clase protegidos por los medios más diversos y por este motivo difíciles de enfrentar. A menudo las críticas que desprecian el opúsculo no se detienen lo suficiente en esta tensión que atraviesa todo el texto ${ }^{13}$.

La hipótesis de la autoría de un ateniense o extranjero que explica a otros la situación puede convivir, en alguna medida, con la idea del texto como un informe de inteligencia y tendría como respaldo ciertos elementos que evalúan la posición de Atenas frente a un conflicto ${ }^{14}$. El Viejo Oligarca es en este sentido un maestro cuidadoso y paciente que comienza declarando su acuerdo ante esta animadversión y la fundamenta en la oposición entre valiosos e inútiles (I.4), para pasar paulatinamente a acompañar a su lector en un viaje a las entrañas de la democracia que desdibuja los golpes iniciales. En algún sentido, a medida que avanza el texto va cediendo la beligerancia, en tanto se comprende paulatinamente el punto de vista de los sostenedores del sistema. No es ruinoso y no es fruto de una crisis, sino un modo de organización cuya lógica interna es comprensible.

En algún sentido, el sistema democrático descansa sobre una visión más optimista sobre la autorregulación de las variantes que llevan al equilibrio, mientras la oligarquía, más dudosa sobre este punto, prefiere confiar en

13 Véase Mársico, Illarraga y Marzocca (2017) y Christodoulos (2017).

14 Véase Rossetti (1975), Marr y Rhodes (2008, pp. 1-15), Mattingly (1997, p. 355). 
pertenencias y saberes específicos. No se trata tanto de abolición de diferencias o sumisión, dado que incluso si se concede que la democracia ateniense incluía una base social más amplia, sus tendencias imperialistas invalidan cualquier "leyenda rosa". Tampoco se trata de un alegre desenfreno, como quiere instalar el Viejo Oligarca y después repite Platón en República, VIII.557c comparando la democracia a una virtual ausencia de sistema político donde cada uno hace lo que quiere según criterios caprichosos, con la irreflexión, dice Platón, de las mujeres y los niños. Basta tener en cuenta la multiplicación de los recodos institucionales burocráticos o recordar el cuidadoso sistema de sorteo como verdadera base del acceso a cargos para captar que lo que varían son los presupuestos metafísicos de cada perspectiva, de modo que donde la democracia piensa que la "masa de lo real" en su permanente discurrir tiende al equilibrio, la oligarquía ve un riesgo de caos que amenaza acabar con todo.

Las consecuencias prácticas del choque entre las dos concepciones, por supuesto, efectivamente produjo colisiones de dimensiones que ameritan que sigamos recordándolas, no porque esas dimensiones sean en sí mismas trágicas, aunque incluyen momentos que lo son, como la matanza de Melos cuyo reflejo reverbera en las Troyanas de Eurípides ${ }^{15}$, sino porque conceptualmente señalan el motivo más profundo de toda crisis política que tiene en el sufrimiento de los cuerpos la expresión tangible del choque entre ontologías incompatibles. La claridad meridiana con que lo expresa la tensión entre los textos antiguos motiva en buena medida la iteración de la vuelta en la mirada.

Como ejemplo de este retorno, que podríamos ilustrar con numerosos ejemplos contemporáneos, recordemos la intuición foucaultiana que conduce a la identificación de dos condiciones paradojales que sugieren que la democracia no trastabilla por la crisis provocada por la acción u omisión de sus agentes, sino por una grieta estructural de la asociación entre isegoría y parrhesía, elementos que constituyen, siguiendo a Polibio, su núcleo ${ }^{16}$ : la veridicción riñe con la isegoría, ya que no todos están en condiciones de enunciarla, pero no puede haber democracia sin veridicción. Esta convivencia condenada al fracaso provoca el deslizamiento de la parrhesía hacia un nuevo terreno que no es el de la política sino el de la filosofía, provocando la crisis terminal de la democracia del s. V a.C. Se trata de una expresión de la variante de la colisión entre modelos democráticos y oligárquicos y refleja, por tanto, una parte de las fricciones en juego.

Consideremos ahora otra fricción de la democracia, en su colisión con el gobierno unipersonal, ya sea en términos de monarquía o de tiranía. Atenas experimentó esta modalidad con algunas interrupciones, entre el 561 y 510

15 Véase Plácido (2006, pp. 817-822).

${ }^{16}$ Polibio, II.38.6 y Gouv., 68-69. Véase Terrel (2010), Moreno Pestaña (2013, pp. 509-532; 2019, p. 532) y Szakolczai (2013, pp. 177-183).

Araucaria. Revista Iberoamericana de Filosofia, Política, Humanidades y Relaciones Internacionales, año $23, \mathrm{n}^{\circ} 46$. Primer cuatrimestre de 2021. Pp. 71-84. ISSN 1575-6823 e-ISSN 2340-2199 https://dx.doi.org/10.12795/araucaria.2021.i46.04 
a.C., bajo el gobierno de Pisístrato y sus hijos Hiparco e Hipias, desplazado este último por una alianza entre los Alcmeónidas y Esparta. Así como hay un relato que ve la democracia como superación de la oligarquía, hay otro que la considera una salida a la crisis producida por la tiranía. Clístenes, en efecto, fue arconte bajo el gobierno de Hipias y habría tramitado este pasaje. El peso de la evaluación de Heródoto seguramente impulsó este relato, que no acompañaron, por ejemplo, ni Cimón ni Aristóteles ${ }^{17}$. La victoria sobre la tiranía acercaría las posiciones de la aristocracia y los pobres: éstos abandonan sus pretensiones de reforma agraria y ceden el arcontado y los altos cargos militares a la aristocracia, mientras ésta promete una política exterior que los favorezca. La democracia estabilizaría el sistema de alianzas que permitiría la protección de los intereses de todos los grupos incluidos los oligárquicos, de modo tal que la crisis se vuelve el costo de la dinámica que mantiene a raya los intentos de gobierno unipersonal.

La estrategia fue exitosa, en tanto un sentido inicialmente ausente del término túrannos se volvió usual y se lo ligó a la violencia y la ilegalidad ${ }^{18}$. Esta asociación forma parte de una operación de sentido y no debe olvidarse que Grecia se preparaba para el gobierno unipersonal que la unificaría y catapultaría a la conquista de Asia. Con esto queremos decir que durante la época clásica los gobiernos unipersonales estaban extendidos en las colonias y Jenofonte se dedica en el Hierón a revisar las potencialidades de este modelo, de modo tal que no era, en el medio de la crisis ateniense, una pieza de museo sino una posibilidad abierta que las otras dos variantes intentaban acallar.

El Hierón de Jenofonte ofrece precisamente una impugnación de la asociación entre tiranía y violencia que en muchos aspectos recuerda la estrategia del Viejo Oligarca: parte de un retrato del tirano de Siracusa atravesado de la infelicidad derivada del odio que produce en los ciudadanos e incluso en su círculo íntimo para transformar este cuadro en un programa de gobierno que hace del gobernante un hombre amado por sus súbditos y de la ciudad un ámbito próspero. En efecto, Hierón se siente preso del temor a ser asesinado y rodeado solamente de hipocresía. Se ve como un sujeto miserable que, casi como en la recomendación del sileno en el Eudemo de Aristóteles, debería preferir no haber nacido o, si nació, morirse pronto ${ }^{19}$. El propio Hierón sostiene que si a alguien le conviene ahorcarse, ése es el tirano (VII.13). En respuesta, Simónides, un Sócrates jenofonteo, ofrece una especie de sintético programa de tranformación política. Simónides recomienda a Hierón que comience por

\footnotetext{
${ }^{17}$ El primero las consideró aristocráticas y el segundo más democráticas que las de Solón, pero no ejemplo abierto de este sistema.

18 Parker (1998, pp. 145-172), McGlew (1993) y Cawkwell (1995, pp. 73-86).

19 Véase Plutarco, Consolación a Apolonio, 27, 115b-c (Frag. 44 Rose). Sobre el Eudemo, véase Bos (1984, pp. 19-51) y (2003, pp. 238 ss.).
} 
enriquecer a su entorno, porque con eso se enriquece a sí mismo, y a su ciudad, porque sus allegados lo fortalecen.

Hay que entender que sus allegados conforman su grupo político de acción, mientras la ciudad constituye su base territorial, es decir el ámbito de actores políticos que le responden y por tanto el andamiaje que cimenta su poder. Con su fuerza política ordenada y su territorio asegurado, se recomienda inmediatamente atender a la cuestión geopolítica y recabar aliados que protejan su posición global. En esta especie de gradación entre política "partidaria", nacional e internacional, la garantía última no es la fuerza sino el buen gobierno que capte el apoyo de cada vez más voluntades. El terror que paralizaba y hacía miserable la vida de Hierón se desvanece en un final en el que Simónides aventura en \# XI.15 que si no se aparta de los ejes de multiplicación del bienestar y administración de justicia es posible mantener en aumento la masa popular que apoya la gestión de gobierno.

La felicidad, como extremo de lo personal, se da con la condición de una transformación hacia lo público, y adviene de un modo que supera la felicidad privada porque escapa a la envidia en tanto produce felicidad para otros. La respuesta a la pregunta respecto de la calidad de la felicidad que alcanzan gobernante y súbdito queda entonces respondida de dos modos: en la versión hieroniana, el gobernante instalado en lo privado queda despedazado por una maquinaria que lo excede y lo destroza; en la versión simonidiana, el gobernante instalado en lo público se convierte en el eje de transformación social y supera con ello cualquier proyección del ámbito de lo privado. El político persigue el poder para mejorar la vida de sus conciudadanos y, en este mismo movimiento, mejorar también su propia existencia con la potenciación de todas sus relaciones humanas, de un modo que justifica los esfuerzos y sinsabores de la gestión del poder. Los ecos platónicos son llamativos y abren una vía interesante de exploración sobre la comunión de opiniones sobre este ámbito en el círculo socrático.

El punto se vuelve especialmente interesante dado que en la confrontación del gobierno unipersonal con la democracia y la oligarquía a la vez se juega el destino de dos protagonistas de la crisis ateniense del s. V a.C. que a menudo han quedado separados a pesar de su vínculo. Nos referimos a Teramenes y Sócrates. El primero fue apodado de "coturno", el zapato que calza en los dos pies, para resumir sus cambios de posición política. Lo que esconde el apodo es que Teramenes no se movía siempre hacia lugares cómodos, sino, al contrario, sus posiciones críticas terminaron convirtiéndolo en un enemigo de Critias. Tras su negativa a la ejecución de metecos fue condenado a tomar la cicuta en 404 a.C. Según relatan las fuentes, Sócrates intentó rescatarlo y se encontró con la negativa de Teramenes, que prefirió asumir la condena ${ }^{20}$. El episodio de la

${ }^{20}$ Véase Perrin (1904, pp. 649-669) y Pesely (1988, pp. 31-33).

Araucaria. Revista Iberoamericana de Filosofia, Política, Humanidades y Relaciones Internacionales, año $23, \mathrm{n}^{\circ} 46$. Primer cuatrimestre de 2021. Pp. 71-84. ISSN 1575-6823 e-ISSN 2340-2199 https://dx.doi.org/10.12795/araucaria.2021.i46.04 
negativa de apresar a León de Salamina por parte de Sócrates que relata Platón en Apología, 32c-d, indicada del mismo modo por el gobierno de los Treinta, y la negativa a escapar con Critón -o con Esquines, en otras versiones-, configuran una serie de iteraciones llamadas a hacer de Sócrates un nuevo Teramenes. Uno es ejecutado por la oligarquía y el otro por la democracia, precisamente porque en muchos puntos encarnan la oposición a ambas en una apelación que resuena en las obras de Jenofonte y Platón.

\section{Corolarios}

No nos detendremos aquí en la colisión entre oligarquía y gobierno unipersonal, dado que nuestro tema es la crisis de la democracia, pero la criba de nociones afecta todo el espectro. Más todavía, la crisis alienta un más allá de la tríada de Heródoto, de modo que comienzan a florecer en esta época con vivacidad distintas exploraciones de modelos alternativos, donde resuenan especialmente las variantes irrealistas que atraviesan las obras de Aristófanes, Isócrates, Antístenes y Platón. Los textos de Asambleistas, el Busiris, la comunidad de cíclopes y República forman parte de un mismo movimiento de edificación de modelos posibles que continúa las exploraciones que hemos visto acerca de los tres sistemas básicos. Los aspectos perturbadores en riesgo constante de disolución resultan la condición de posibilidad para pensar la superación de estructuras que resultan rápidamente anquilosadas e intentar sin denuedo el diseño de nuevos parámetros generales. En este sentido, la crisis ateniense ilustra una instancia de puesta en cuestión de lo dado y de creación de universos posibles en el horizonte de un movimiento de inestabilidad permanente que nos resulta particularmente cercano y continua su curso en nuestros tiempos, tan griegos. 


\section{Referencias bibliográficas:}

Bos, A. (1984). Aristotle's Eudemus and Protrepticus: Are they really two different works? Dionysius, 8.

Bos, A. (2003). The Soul and its Instrumental Body: a reinterpretation of Aristotle's philosophy of living nature. Leiden: Brill.

Cawkwell, G. (1995). "Early Greek tyranny and the People". Classical Quarterly, 45.1.

Chantraine, P. (1968). Dictionnaire étymologique de la langue grecque. Histoire des mots. Paris: Klincksieck.

Cherniss, H. (1953). "The History of Ideas and Ancient Greek Philosophy", Studies in Intellectual History. Boston: Johns Hopkins Press.

Christodoulou, P. (2017) "Thucydides Philosophistoricus: The Way of Life of the Historian", Araucaria, 37.

Derderian, K. (2001). Leaving words to remember: Greek mourning and the advent of literacy. Leiden: Brill.

Glassman, R. (2017). The Origins of Democracy in Tribes, City-States and Nation-States. Dordrecht: Springer.

Gottwald, N. (2001). The politics of ancient Israel. Louisville: Westminster John Knox Press.

Isakhan, B. (2007). "Engaging" primitive democracy": Mideast roots of collective governance". Middle East Policy 14.3.

Jacobsen, T. (1943). "Primitive democracy in ancient Mesopotamia". Journal of Near Eastern Studies 2.3, 159-172.

Loraux, N. (1986). The invention of Athens: The funeral oration in the Classical City. Cambridge, CUP.

Marr, J. y Rhodes, P. (2008) The Old Oligarch, The Constitution of the Athenians attributed to Xenophon, London: Oxbow.

Mársico, C. (2011). "Ejes para pensar lo griego". En: C. Mársico (Ed.), Polythryleta. Sistemas explicativos y mutación conceptual en la antigua Grecia, Buenos Aires: Rhesis.

Mársico, C., Illarraga, R. y Marzocca, P. (2017). Jenofonte, Constitución de los espartanos y Hierón; Pseudo-Jenofonte, Constitución de los atenienses. Buenos Aires: UNQui.

Martin, M., Snell, D. (2005). "Democracy and freedom". En: D. Snell (Ed.), A Companion to the Ancient Near East, Oxford: Blackwell.

Mattingly, H. (1997). "The Date and Purpose of the Pseudo-Xenophon Constitution of Athens". Classical Quarterly, 47.2.

McGlew, J. (1993). Tyranny and Political Culture in Ancient Greece. London: Cornell University Press. 
Moreno Pestaña, J. (2013). "Isegoría y parresia: Foucault lector de Ión". Isegoría, 49.

Moreno Pestaña, J. (2019). Retorno A Atenas: La democracia como principio antioligárquico, Madrid, Siglo XXI.

Parker, V. (1998). "Túrannos. The semantics of a political concept from Archilochus to Aristotle". Hermes, 126.

Perrin, B. (1904). "The Rehabilitation of Theramenes". The American Historical Review 9.4.

Pesely, G. (1988). "Socrates' Attempt to Save Theramenes". The Ancient History Bulletin 2.

Plácido, D. (2006). "Vencedores y esclavos: Las Troyanas de Eurípides". Koinòs lógos. Homenaje al profesor José García López, Murcia.

Rodríguez Guerra, R. (2011). "Las reformas de Clístenes: cuándo, cómo y por qué. En torno a los orígenes de la democracia". Laguna: Revista de Filosofia 29, 149-168.

Rossetti, L. (1975). "Alla ricerca dei logoi sokratikoi perduti (I-III)". Rivista di Studi Classici [Torino], XXII-III, 102.

Schemeil, Y. (2000). "Democracy before democracy?". International Political Science Review 21.2: 99-120.

Stockwell, S. (2011). "Before Athens: Early popular government in phoenicia and Greek city-states", The Secret History of Democracy. London: Palgrave Macmillan.

Szakolczai, A. (2013). Comedy and the Public Sphere: The Re-birth of Theatre as Comedy and the Genealogy of the Modern Public Arena. London: Routledge.

Terrel, J. (2010). Politiques de Foucault. Paris: PUF.

Van De Mieroop, M. (2013). "Democracy and the Rule of Law, the Assembly and the First Law Code". The Sumerian World, 277-89.

Vilanou, C. (2006). "Historia conceptual e historia intelectual". Ars Brevis 12, 165-190.

Wolf, C. (1947). "Traces of primitive democracy in ancient Israel". Journal of Near Eastern Studies 6.2, 98-108. 